\title{
Raper Robot Local Network Using TQS Protocol for Land-to-Underwater Communications
}

\author{
Addie Irawan, Mohammad Fadhil Abas, and Nurulfadzilah Hasan \\ Faculty of Electrical and Electronics Engineering, Universiti Malaysia Pahang, Pekan, Malaysia
}

https://doi.org/10.26636/jtit.2019.125818

\begin{abstract}
This paper presents a model and an analysis of the Tag QoS switching (TQS) protocol proposed for heterogeneous robots operating in different environments. Collaborative control is topic that is widely discussed in multirobot task allocation (MRTA) - an area which includes establishing network communication between each of the connected robots. Therefore, this research focuses on classifying, prioritizing and analyzing performance of the robot local network (RLN) model which comprises a point-to-point topology network between robot peers (nodes) in the air, on land, and under water. The proposed TQS protocol was inspired by multiprotocol label switching (MPLS), achieving a quality of service (QoS) where swapping and labeling operations involving the data packet header were applied. The OMNET++ discrete event simulator was used to analyze the percentage of losses, average access delay, and throughput of the transmitted data in different classes of service $(\mathrm{CoS})$, in a line of transmission between underwater and land environments. The results show that inferior data transmission performance has the lowest priority with low bitrates and extremely high data packet loss rates when the network traffic was busy. On the other hand, simulation results for the highest $\mathrm{CoS}$ data forwarding show that its performance was not affected by different data transmission rates characterizing different mediums and environments.
\end{abstract}

Keywords-class of service, land-to-underwater communications, robot local network, tag switching.

\section{Introduction}

Heterogeneous robot communication or multirobot networking is conducted mainly via the Internet. There are many issues related to establishing robot local networks (RLN) for collaboration purposes, and they need to be resolved in order to achieve specific objectives, with a particular emphasis placed on dependability, safety and security of the system. To enable collaboration between robots, two elements need to be defined, i.e. the communication protocol and the collaborative procedures. Undoubtedly, an efficient protocol is essential for effective decentralization of the distribution of data between the robots.

Researchers have taken several approaches to improve communication between heterogeneous robots. Some of them rely on the communication protocol, while others use the intelligent control technique to improve the performance of the system. There is also a hybrid approach which combines both these techniques. Stamatescu et al. described the communication protocol by applying the cognitive radio (CR) scheme, i.e. by exploiting the time, frequency and spatial stream of the wireless environment. According to the testing results, they claimed that the communication reliability at each hierarchical level increased [1]. Another approach consists in applying a formal taxonomy to the allocation of tasks to a mobile robot, as proposed by Gerkey and Mataric [2]. This method is further improved by Korsah et al. with their proposed iTax, a taxonomy addressing interrelated utilities and constraints via a combination of optimization methods and operation research. This method is based on the recognition that the key distinguishing factor between different types of multirobot task allocation (MRTA) problems is the degree of interdependence of agent-task utilities [3].

Adaptation of intelligent systems is also becoming an approach that is favored in multirobot collaborative control and communication. Zhang et al. applied an adaptive fuzzy logic in tackling MRTA reliance on the intuitionistic fuzzy set theory [4]. Similarly, Cheng et al. proposed a linear temporal logic (LTL) which optimizes path planning by using the formation control feedback mechanism [5]. Power is another constraint that needs to be considered while establishing a collaborative robotic system. Moreover, there is a tradeoff between the number of nodes that can be deployed in the mission and the density of information that can be exchanged. Bano et al. in [6] explored these constraints and proposed a random waypoint mobility model in a mobile ad-hoc network (MANET). This system was tested with a robot group comprising 5-6 collaborating robotic nodes and the results showed that it was better than the Manhattan mobility model. MANET is also implemented by Kulla et al. for a real-time emergency scenario of moving the multirobot (nodes) indoor [7]. Bandwidth sharing is also possible for an MRTA communication system using resource controller (RC) and aggregate resource controller (ARC) management techniques [8].

Classification and aggregation also offers a vast potential that may be explored in robot-to-robot or human-to-robot communication, and mainly in enhancing reliability per- 
formance and in ensuring priority for different types of data exchanged over the Internet. Automation and robotics place a greater emphasis on the physical layers, with realtime data and control area network (CAN) serving as the primary platform. A virtual private network is available in the Internet protocol (IP) version, such as the virtual private network (VPN) used for long distance and indoor remotely controlled mobile robots [9], [10]. However, the problem is still an issue in the case of communication between mobile robots (swarm scenario).

The present research proposes the application of tag switching inspired by multiprotocol label switching (MPLS) [11], as a data carrying technique for an RLN operating in a different environment. A modular network testbed in $\mathrm{C}++$ $(\mathrm{OMNeT}++)$ [12], i.e. a discrete event simulator, was used to develop a logical RLN model to perform a case study focusing on multirobot communication in a different environment. The nodes in the RLN were programmed with the Tag QoS switching (TQS) protocol proposed, and were considered to be robots (moving nodes). Data forwarding performance, expressed as the percentage of packets lost, average edge-to-edge access delays, as well as throughput, was verified by comparing one line of transmission between the edges of robots in a different environment.

\section{Modeling of a Local Multirobot Network with Tag Switching Protocol}

\subsection{Case Study Involving Land-to-underwater Communication}

For the case of multirobot communication involving different environments, e.g. in the land-to-underwater scenario, the robots may be flying in the air, may be submerged under water or may be placed on the ground, as shown in Fig. 1. Underwater communications have limitations in terms of distance and bandwidth. For example, optical wave transmission requires high precision in pointing narrow laser beams and is affected by scattering, although is resistant to high attenuations [13]. On the other hand, electromagnetic waves are also limited to short distance with the highest frequency at about $2.4 \mathrm{GHz}$ for $250 \mathrm{Kbps}$, according to

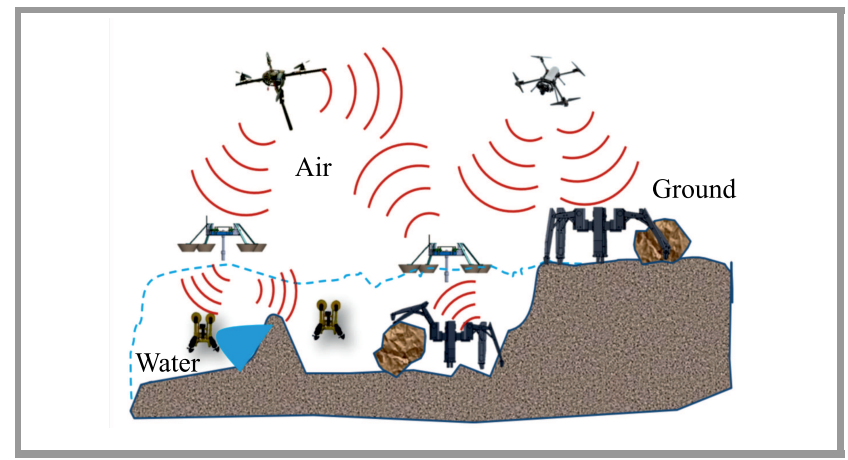

Fig. 1. Example of a heterogeneous RLN topology for land-tounderwater communications. the IEEE 802.15.4 standards [14]. Alternatively, data signal may be also propagated in conductive salty water by using radio frequency (RF), but only at extra-low frequencies $(30-300 \mathrm{~Hz})$ that may require large antennas and high transmission power [15]. Several attempts have been made to enhance the speed and throughput of transmission, such as a routing technique relying on the surface of the water for underwater communications [16], as well as using the water surface relay to increase the overall transmission speed [17].

Therefore, this study investigates and analyzes data forwarding and switching/routing performance for the landto-underwater communication scenario, using the proposed TQS protocol. It emphasizes the RLN topology in which robots are considered to be dynamic nodes of the switches, or routers for data transceivers. This study neglects all salt- and tide-related factors, as well as noise present in both mediums. The analysis focuses on logical data transmission implications and on the dynamic changes in data transmission rates experienced when the packet of data enters the water using the proposed protocol. Moreover, the focus is placed mainly on data the forwarding period and on traffic management. Some of the switching/router models were programmed to operate at low data transmission rates $(10-100 \mathrm{Kbps})$ to represent nodes in the underwater environment, while other were programmed for speeds of up to $1 \mathrm{Gbps}$ to represent nodes on land/in the air.

\subsection{Tag-QoS Switching Protocol}

The TQS protocol is suited for a network topology with dynamic nodes, such as RLN, as it is inspired by MPLS. MPLS was released by Cisco System in 1998 and started to gain popularity in IP deployment for wide area networks (WAN) and metropolitan area networks (MAN) [18], [19] in 2000. MPLS allows tunnel routing known as label switched paths (LSP), where a tunnel is characterized by a path in the network and by a reserved bandwidth [20], [21]. This protocol belongs to layer 2.5 in the open systems interconnection (OSI) model [22]. It improves both layers 2 and 3 by providing fast switching and reliable routing. Moreover, this protocol is bonded to an IP network as an extra header that involves the Internet service provider (ISP) area [23], [24]. However, the dynamic tagging and stacking methods used in MPLS have the potential to be deployed in a small-scale local network and data communication scheme, such as RLN shown in Fig. 1. The label swapping concept in MPLS [25] enables dynamic establishment of tunnels that depends on traffic demand. The tunnels are opened based on aggregation, classification and prioritization of communication between peer robots. MPLS also enables network virtualization through the labeling or tagging method, in order to create virtual and physical layers [26] that leverage the implementation of energy-aware traffic engineering [27].

The TQS protocol proposed is applied in the same manner as the MPLS label stack entry (LSE) shown in Fig. 2, but with specific label value calculations which involve both the 
indication of the LSP and the differential service (DiffServ). The calculation also includes a flow aggregate that requires network traffic to be marked and conditioned at the edges of the network, ensuring a different treatment for each of the tagged packets. The label value in TQS-LSE is:

$$
L=[\alpha(N+1)]+P,
$$

where $\alpha$ is the definite positive gain used for simple indication and for reducing conflicts in the tagging process. $N=1,2, \ldots, n$ is an LSP identification number, expressed as an integer, which represents the type of incoming data e.g. video or voice streaming. $P=0,1,2, \ldots, n$ is denoted as a sub-LSP identification number for the bandwidth that is generated by the packet index at the edge of the node $I$ when the number of channels for the $D$ group of bandwidths satisfies the condition $I>D-1$. The $P$ value can be obtained from:

$$
P=\left\{\begin{array}{ll}
P=0 & I>D-1 \\
P=I & D \leq D-1
\end{array} .\right.
$$

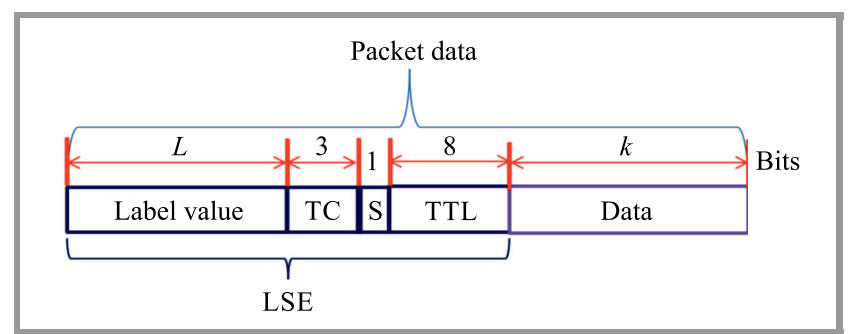

Fig. 2. LSE as a data tag in the Tag QoS switching protocol proposed in an RLN data distribution scheme.

As far as RFC 3270 is concerned, the experimental bits (EXP), renamed as traffic class (TC) bits [28], are used to encode and aggregate all per-hop behavior (PHB) bits from the data header to LSE [29]. In addition to that, three behaviors aggregating bits (BA) were used to encode with the DiffServ code point (DSCP) from the data header [30]. DSCP defines drop precedence in each type of class of services $(\mathrm{CoS})$ for each data packet. MPLS-QoS encoding provides inferring $\mathrm{CoS}$ and drops precedence information from the data header to LSE. The bottom of stack $(\mathrm{S})$ bit for the last entry of the label stack indicator and time-tolive (TTL) bits, as shown in Fig. 2, are the standard bit in the MPLS format, as defined in RFC3032 [11].

\subsection{RLN Topology Model with TQS Protocol}

For modeling and simulations relying on the proposed protocol, the nodes of switches/routers (robots) were categorized into two sections: tag edge mobile robot (TER) and tag switching robot (TSR). These switches/routers are the primary entities in RLN acting as transceivers for edges and switching, respectively, according to the TQS protocol data forwarding. As shown in Fig. 3, for simulation and analysis purposes, the RLN was modeled with several

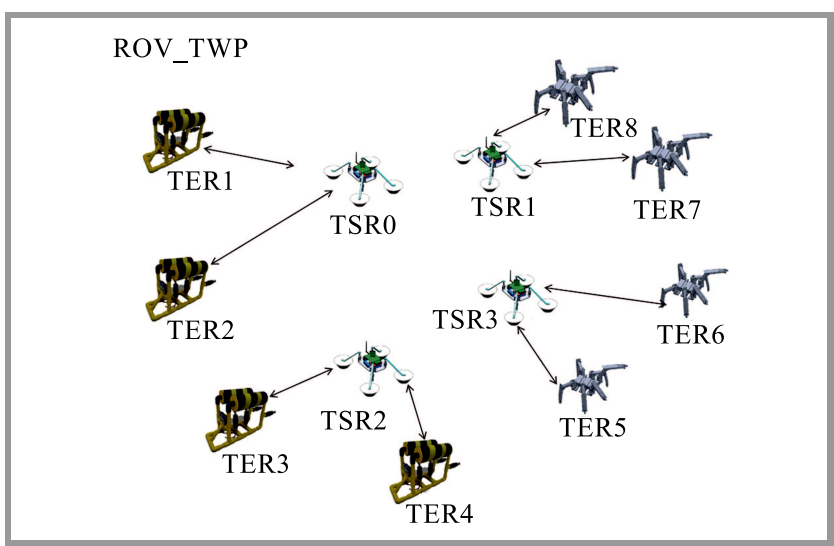

Fig. 3. RLN model topology for simulation and analysis in the OMNeT++ graphical runtime environment.

TERs and TSRs in OMNeT++. Here, the TER module was programmed to generate a raw/unlabeled data packet. The system consists of three sub-elements which are categorized as wire switch module (WSM), buffer switch module (BSM), and bandwidth switch module (BandSM), as pictured in Fig. 4. The usage of WSM in the TER module is the main feature that differentiates it from the TSR (Fig. 5). The switching process is applied when the label was swapped in the TSR instead of WSM, as shown in Fig. 6. The incoming tagged packets are buffered and then passed to the tag switching module (TSM) to swap the tag or label for the next hop bandwidth in BandSM. The swap process depends on the information label map (ILM) that has been programmed in the forwarding information table (FIT) of TSR (Fig. 6). Label value will always be swapped or replaced with a different number for the next hop bandwidth. The same goes for TTL as S bit values, they are also continuously updated. On the other hand, the information about bandwidth assignment is extracted in BandSM from the inverse calculation of Eq. (1) to get the information about the LSP and sub-LSP switched to the next hop of peer robot/node. BandSM, either in TER or TSR, is programmed to control the per-flow threshold according to the proposed bandwidth assignment scheme (BandAS), as presented in Table 1. The peak data rate (PDR) for premium/expedited forwarding $\mathrm{CoS}$, or the committed data rate (CDR) for Olympic/assured forwarding $\mathrm{CoS}$ will discard the incoming data packets whenever the threshold is reached.

For the TER model, the process of forwarding the equivalent class (FEC) [11] to the next hop label forwarding entry (FTN) is applied in WSM as a labeling process, whereby the untagged data packet destination address is screened for the labeling process (Fig. 7). Initially, the data are generated with source address bits, destination/group address bits and hop limit values that are the same as those of packet data with the IP address. Moreover, the DSCP code in the generated packet data was aggregated to request the FEC code from the programmed FIT. Then, the label value with L bits (here 10 bits) was obtained (Fig. 7). The hop limit value and the DSCP code were then encoded to the TTL 


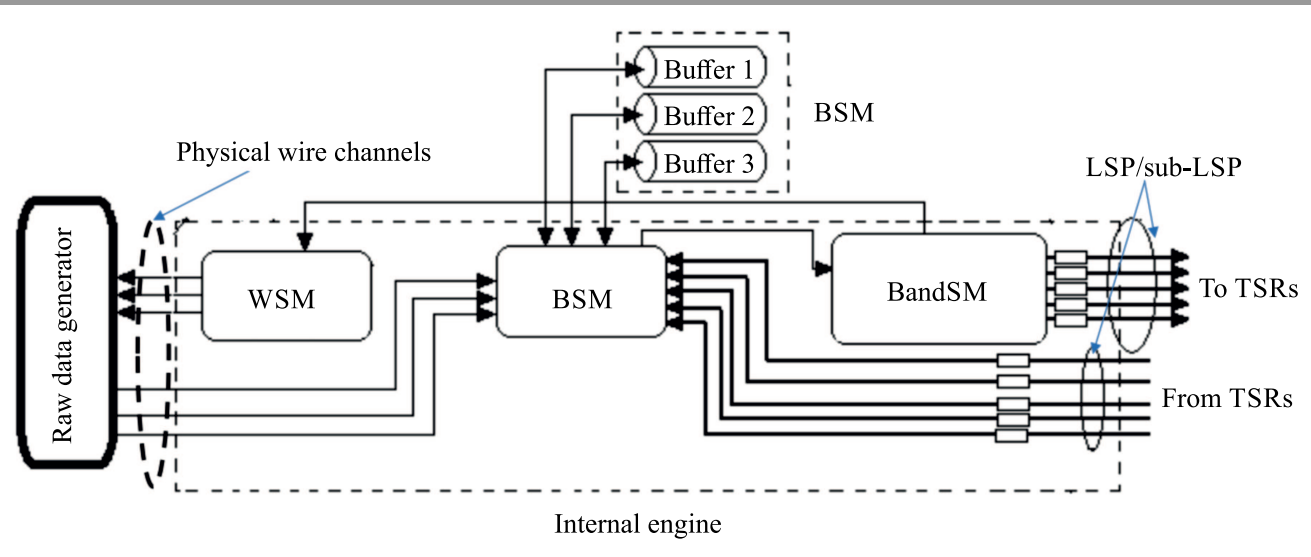

Fig. 4. TER model system engine with the TQS protocol proposed.

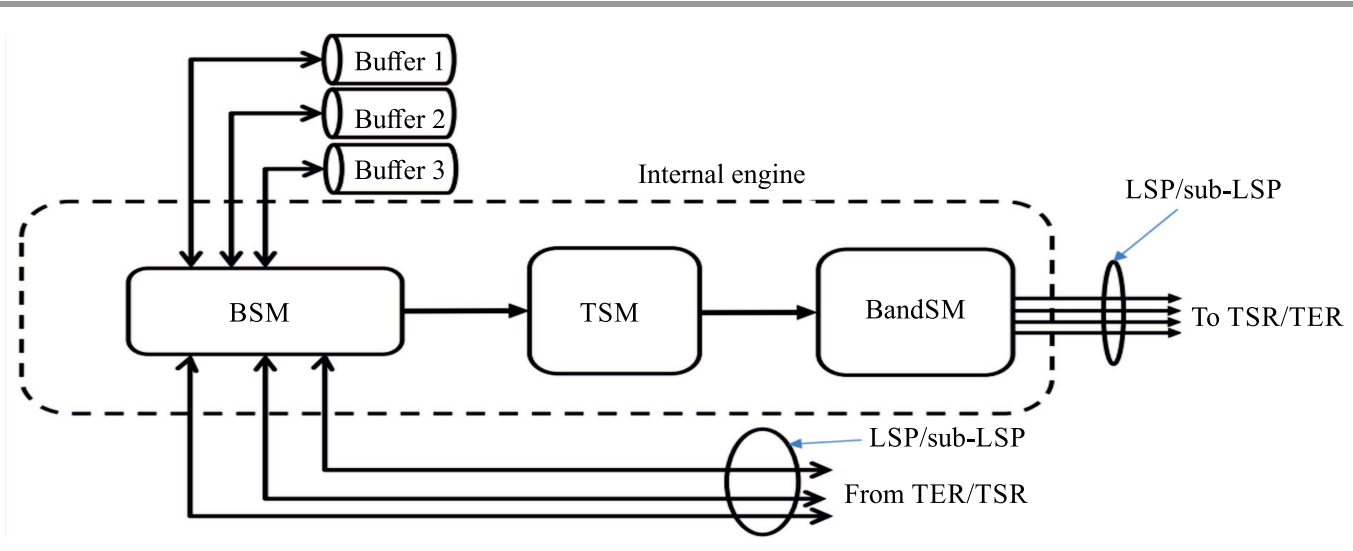

Fig. 5. TSR model system engine with the TQS protocol proposed.

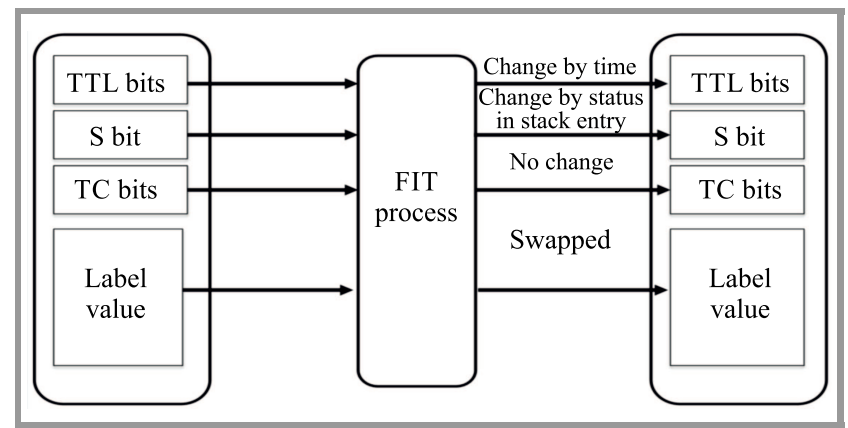

Fig. 6. Swapping process in BandSM.

Table 1

Bandwidth assignment scheme (BandAS)

\begin{tabular}{|c|c|c|}
\hline \multicolumn{2}{|c|}{ Class of services } & Channel bit rate \\
\hline \multicolumn{2}{|c|}{ Premium } & \multirow{2}{*}{$100 \%$ maximum bandwidth } \\
\hline \multirow{3}{*}{$\frac{\stackrel{.}{\frac{D}{2}}}{\frac{2}{0}}$} & Gold & \\
\hline & Silver & $80 \%$ maximum bandwidth \\
\hline & Bronze & $60 \%$ maximum bandwidth \\
\hline \multicolumn{2}{|c|}{ Best effort } & $40 \%$ maximum bandwidth \\
\hline
\end{tabular}

field and TC bits, respectively, using the MPLS-QoS [29] encoding method. The CoS for the applied QoS is determined according to the RFC 2597 draft, in which gold, silver, and bronze $\mathrm{CoS}$ of the Olympic $\mathrm{CoS}$ are applied [31].

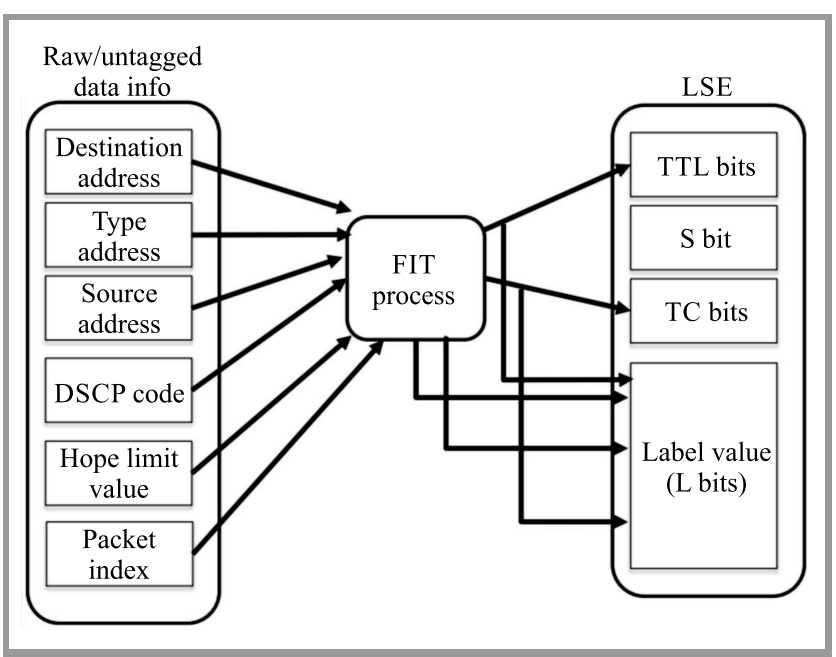

Fig. 7. FTN process for an unlabeled data packet in TM inside the TER model. 
The drop precedence process is also conducted differently for all sub-CoS in the Olympic CoS. Hence, the channel bit rate was defined differently for the sub-CoS of the Olympic $\mathrm{CoS}$ in BandAS, as shown in Table 1. Gold CoS was the lowest drop precedence, and bronze $\mathrm{CoS}$ was the highest. With reference to the MPLS-QoS queuing process [29], as well as RFC2597, WSM in each TER and BandSM in TSR was programmed to perform marking through the labeling process on the untagged data packet. Data packet with low drop precedence was marked as a low priority packet to be discarded instead of the data packet with high drop precedence in BSM.

Table 2

Buffer assignment scheme

\begin{tabular}{|c|c|}
\hline Buffer & Capacity (buffer length) \\
\hline \hline B0 & $20 \% \times$ maximum buffer length \\
\hline B1 & $40 \% \times$ maximum buffer length \\
\hline B2 & Maximum buffer length \\
\hline
\end{tabular}

This first select-and-drop process (before BandAS) occurred when the number of packets in the allocated buffer reached its threshold limit, as allocated in Table 2, concerning the simple buffer assignment scheme [32]. The TC bit, as shown in Fig. 2, is an indicator for allocating the data to the particular buffer channel. The first-in-first-out (FIFO) principle was used in the BMS queuing process, where B0 and $\mathrm{B} 2$ for each TER/TSR were programmed to use the tail drop procedure, and B1 was programmed to use the random early detection (RED) procedure [33], mainly for Olympic CoS.

\section{Simulation and Performance Analysis}

Simulation and analysis of the RLN model were conducted and set up with the proposed TQS protocol, as shown in Fig. 3. The setup protocol was considered done in this simulation study, and all nodes were established with the least cost routing. The analysis relied on the same QoS class as-

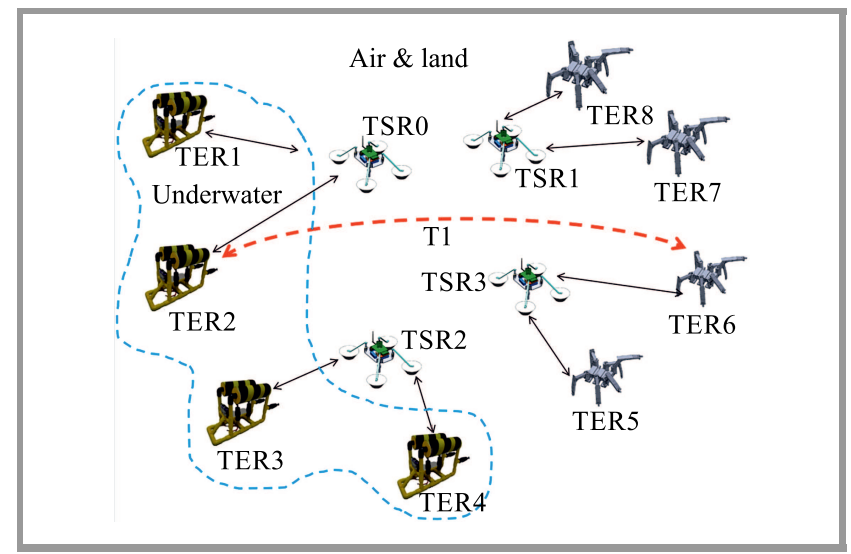

Fig. 8. Analysis notification for simulation purposes and analysis in the modeled RLN. sessment program used in the model as applied in BandAS: premium, Olympic and best effort CoS. The analysis was performed by determining $\mathrm{T} 1$ as a focus line for performance evaluation, with other lines serving as disturbances for T1, as shown in Fig. 8. Moreover, TER1, TER2, TER3 and TER4 were modeled with proper underwater acoustic conditions, where the data rate for receiving/transmitting data packets equaled between 10 and $100 \mathrm{kbps}$. On the other hand, the TSR models were assumed to be positioned on the surface of the water as floating nodes that provided a link between TERs in the air/on land and under water. Communication between TSRs was modeled using ad-hoc communications, with arrows appearing when a link exists between individual peer nodes.

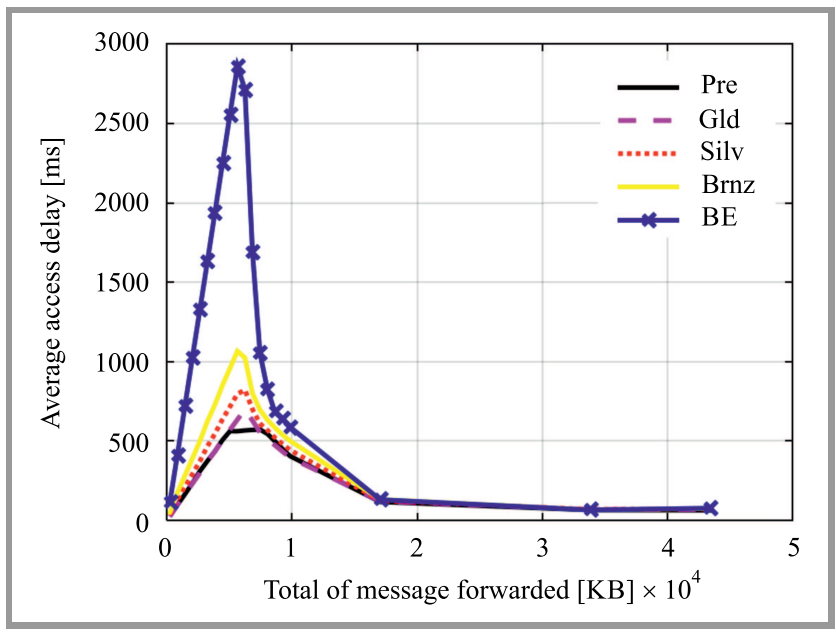

Fig. 9. Average access delay versus total of data packet streaming on $\mathrm{T} 1$.

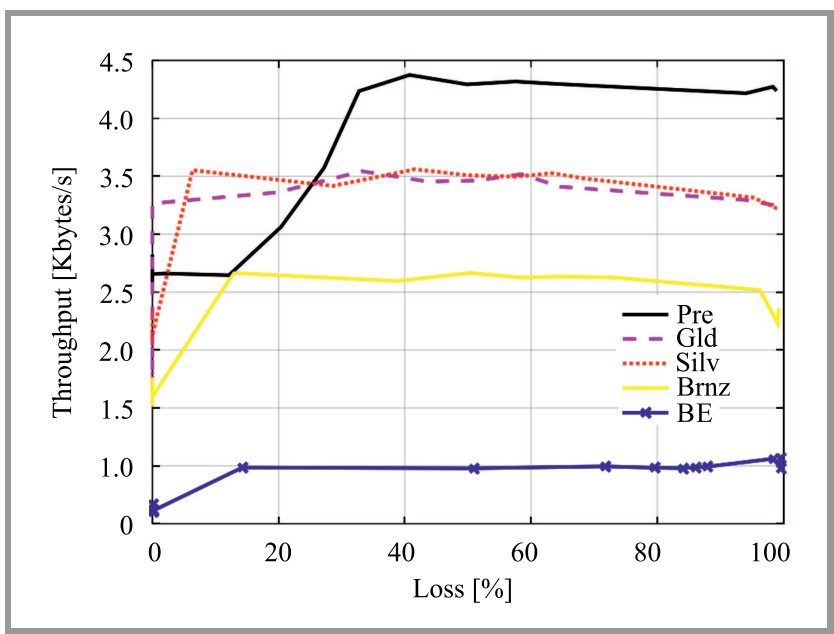

Fig. 10. Throughput versus percentage of data packet loss on $\mathrm{T} 1$.

The results for $\mathrm{T} 1$ data forwarding performance are presented in Figs. 9-12, with the data packet size randomly generated between 250 bytes and 1.5 Kbytes per packet. The results show a different performance of network parameters for different CoS: premium CoS (Pre), best effort (BE), and Olympic CoS members, namely gold $\mathrm{CoS}$ (Gld), 
silver (Silv) and bronze CoS (Brnz). Overall, Pre is the leader in terms of the performance of the majority of parameters.

Figure 9 shows that the average access delay for Pre was the lowest among all forwarding $\mathrm{CoS}$, even though the BandAS channel bitrate was the same as that of the Gld channel, as shown in Table 1. Generally, the percentage of BE forwarding losses was the highest, whereas Pre forwarding was the lowest in both forwarding states (request and reply). This makes the throughputs for Pre $\operatorname{CoS}$ the highest for T1 communication, as illustrated in Fig. 10. In this simulation, other TERs were run to communicate with each other through the shortest path TSRs to provide traffic disturbance (to make the traffic randomly busy).

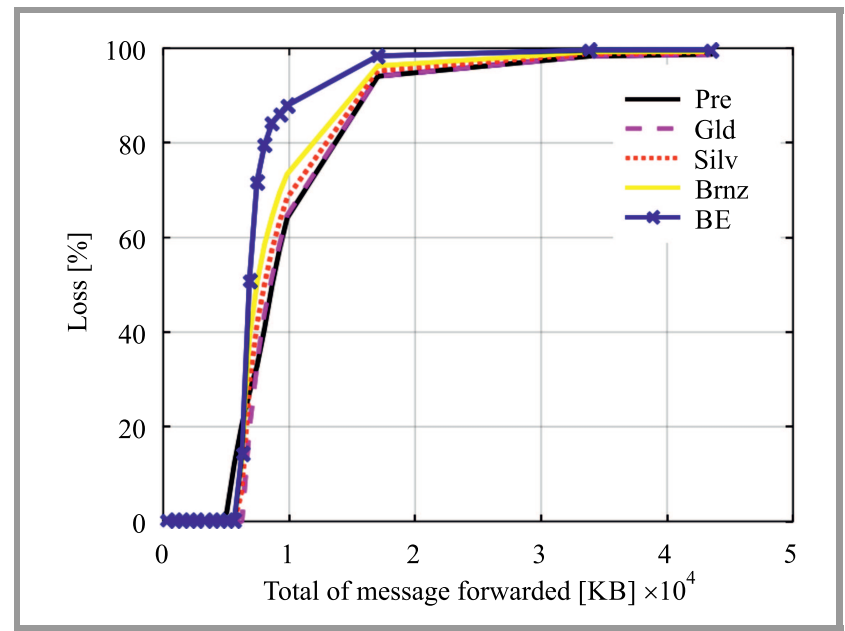

Fig. 11. Data packet loss percentage versus total data streamed on $\mathrm{T} 1$.

As far as the data packet loss percentage rate is concerned, Olympic $\mathrm{CoS}$ and Pre show similar growth trends and are shown in Fig. 11. BE shows the highest rate of data packet loss compared to other scenarios. The results are most evident when the overall data streaming rate on $\mathrm{T} 1$ was between 5000 and 20,000 KB. In this case, the loss percentage rate for $\mathrm{BE}$ is by about $61 \%$ higher than in the case of other $\mathrm{CoS}$ forwarding methods. Before a loss occurs (total of data streaming rate $<35000 \mathrm{~KB}$ ), access delay of Pre forwarding was by about $10 \%$ lower than in each of Olympic CoS forwarding members, and by almost $100 \%$ lower than in the default forwarding or BE. The differences in the performance of individual $\mathrm{CoS}$, with increasing data forwarding rates in T1, are shown in Figs. 9 and 11.

As far as the comparison between access delay and data packet loss, as shown in Fig. 12 is concerned, BE forwarding shows a considerable decrease, as data packet loss rate approaches $100 \%$. However, the average access delay of Pre forwarding is still the lowest and within the acceptable range as the value continues to drop with the increasing data packet loss. Similar results can be observed in the case of Olympic CoS members, where the performance of Gld data forwarding, in terms of access delay, was the best compared to Silv and Brnz, when the data packet loss rate

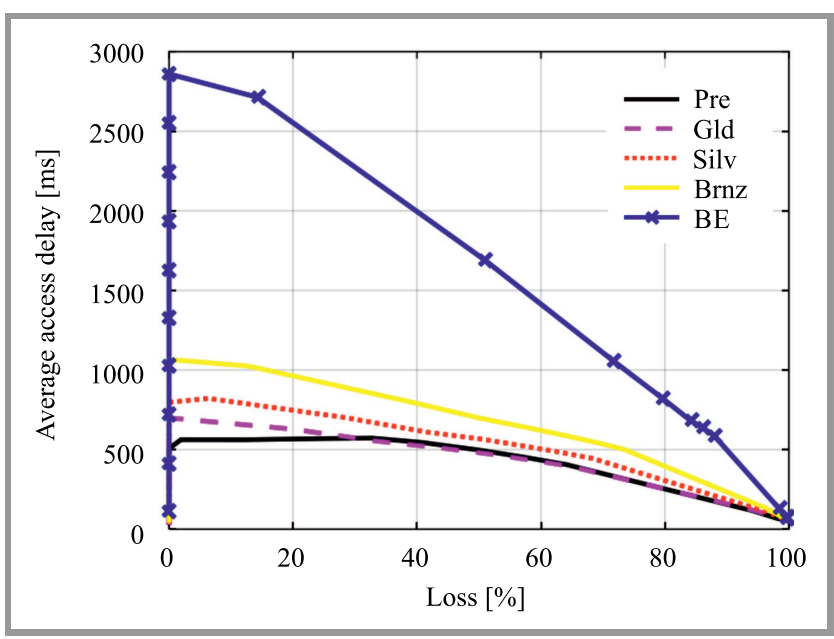

Fig. 12. Average access delay versus data packet loss percentage on $\mathrm{T} 1$.

increased. The results show that both Pre and Olympic CoS have minimal differences in data distribution and switching (Fig. 12). The throughputs to BE CoS forwarding started to decrease when the overall data streaming rate on $\mathrm{T} 1$ increased to more than $300,000 \mathrm{~KB}$.

\section{Conclusion}

The proposed TQS protocol in RLN was modeled and verified. The priority control via $\mathrm{CoS}$ was presented, with prioritization in data routing and switching deployed to achieve different performance outcomes. Pre shows the ability to control the average access delay, although the data packet loss decreased with the increase of the volume of data, since it is the highest priority $\mathrm{CoS}$ as far as minor access delay, high throughput and small data packet loss are concerned. Such a prioritization excludes the factor of different mediums used. Pre and Gld show good reliability for video and voice data forwarding, with the ability to control the number of data packets lost and to achieve a low average access delay, even though the network traffic is busy with handling the number of data packets that increases along with simulation time. The research will continue with the implementation of the proposed TQS protocol in swarm robot RLNs.

\section{Acknowledgment}

This study and research project is funded under the Universiti Malaysia Pahang (UMP) Research Grant for the Centre for Earth Resources Research \& Management (CERRM) of UMP (RDU140351).

\section{References}

[1] G. Stamatescu, D. Popescu, and R. Dobrescu, "Cognitive radio as solution for ground-aerial surveillance through WSN and UAV infrastructure", in Proc. of the 6th Int. Conf. on Electron., Comp. and Artif. Intell. ECAI 2014, Bucharest, Romania, 2014, pp. 51-56 (doi: 10.1109/ECAI.2014.7090210). 
[2] B. P. Gerkey and M. J. Matarić, "A formal analysis and taxonomy of task allocation in multi-robot systems", The Int. J. of Robotics Research, vol. 23, no. 9, pp. 939-954, 2004 (doi: 10.1177/0278364904045564).

[3] G. A. Korsah, A. Stentz, and M. B. Dias, "A comprehensive taxonomy for multi-robot task allocation", The Int. J. of Robotics Research, vol. 32, no. 12, pp. 1495-1512, 2013 (doi: 10.1177/0278364913496484).

[4] L. Zhang, H. Zhong, and S. Y. Nof, "Adaptive fuzzy collaborative task assignment for heterogeneous multirobot systems", Int. J. of Intelligent Systems, vol. 30, no. 6, pp. 731-762, 2015 (doi: 10.1002/int.21725).

[5] C. Cheng, X. Y. Yu, L. L. Ou, and Y. K. Guo, "Research on multirobot collaborative transportation control system", in Proc. Chinese Control and Decision Conf. CCDC 2016, Yinchuan, China, 2016, pp. 4886-4891 (doi: 10.1109/CCDC.2016.7531868).

[6] N. F. Bano, T. Roppel, and I. Gokhale, "Use of mobility models for communication in collaborative robotics", in Proc. 42nd Southeastern Symp. on System Theory SSST 2010, Tyler, TX, USA, 2010, pp. 143-146 (doi: 10.1109/SSST.2010.5442846).

[7] E. Kulla et al., "Real World Emergency Scenario Using MANET in Indoor Environment: Experimental Data", in Proc. 9th Int. Conf. on Complex, Intell., and Software Intensive Syst. CISIS 2015, Blumenau, Brazil, pp. 336-341 (doi: 10.1109/CISIS.2015.49).

[8] P. E. Rybski, S. A. Stoeter, M. Gini, D. F. Hougen, and N. P. Papanikolopoulos, "Performance of a distributed robotic system using shared communications channels", IEEE Transactions on Robotics and Automation, vol. 18, no. 5, pp. 713-727, 2002

(doi: 10.1109/TRA.2002.803460).

[9] Q. Xiao, J. Baojun, D. Xingcheng, Z. Xiaotao, D. Yang, and L. Hui, "A robot remote control system based on VPN and TCP/IP protocol", in Proc. IEEE Int. Conf. on Mechatron. and Autom. ICMA 2008, Takamatsu, Japan, 2008, pp. 285-289 (doi: 10.1109/ICMA.2008.4798767).

[10] K. Kyung Jin, S. Il Hong, K. Sung Hoon, and O. Sang Rok, "A novel real-time control architecture for internet-based thin-client robot; simulacrum-based approach", in Proc. IEEE Int. Conf. on Robot. and Autom., Pasadena, CA, USA, 2008, pp. 4080-4085 (doi: 10.1109/ROBOT.2008.4543838).

[11] E. Rosen, A. Viswanathan, and R. Callon, "Multiprotocol Label Switching Architecture", RFC 3031, IETF, 2001 [Online]. Available: https://tools.ietf.org/ $\mathrm{html} / \mathrm{rfc} 3031$

[12] OMNeT++ Discrete Event Simulator [Online]. Available: https://omnetpp.org

[13] Z. Zeng, S. Fu, H. Zhang, Y. Dong, and J. Cheng, "A survey of underwater optical wireless communications", IEEE Commun. Surveys \& Tutorials, vol. 19, no. 1, pp. 204-238, 2017 (doi: 10.1109/COMST.2016.2618841).

[14] J. Lloret, S. Sendra, M. Ardid, and J. J. P. C. Rodrigues, "Underwater wireless sensor communications in the $2.4 \mathrm{GHz}$ ISM frequency band", Sensors, vol. 12, no. 4, p. 4237-4264, 2012 (doi: 10.3390/s12040423).

[15] D. Pompili and I. F. Akyildiz, "Overview of networking protocols for underwater wireless communications", IEEE Communications Magazine, vol. 47, no. 1, pp. 97-102, 2009 (doi: 10.1109/MCOM.2009.4752684)

[16] M. Dautta and M. I. Hasan, "Underwater vehicle communication using electromagnetic fields in shallow seas", in Proc. Int. Conf. on Elec., Comp. and Commun. Engin. ECCE 2017, Cox's Bazar, Bangladesh, 2017, pp. 38-43 (doi: 10.1109/ECACE.2017.7912875).

[17] H. Yoshida et al., "Study on land-to-underwater communication", in Proc. 14th Int. Symp. on Wirel. Personal Multim. Commun. WPMC 2011, 2011, Brest, France, 2011, pp. 1-5.

[18] B. T. Doshi, R. Nagarajan, G. N. S. Prasanna, and M. A. Qureshi, "Future WAN architecture driven by services, traffic volume, and technology trends", Bell Labs Tech. J., vol. 6, no.1, pp. 13-32, 2001 (doi: 10.1002/bltj.2261).

[19] M. C. Chuah, K. Medepalli, S. Y. Park, and J. Wang, "Quality of service in third-generation IP-based radio access networks", Bell Labs Tech. J., vol. 7, no. 2, pp. 67-89, 2002 (doi: 10.1002/bltj.10006).
[20] O. Klopfenstein, "Rerouting tunnels for MPLS network resource optimization", Eur. J. of Operat. Res., vol. 188, no. 1, pp. 293-312, 2008 (doi: 10.1016/j.ejor.2007.04.016).

[21] S. Ricciardi, F. Palmieri, A. Castiglione, and D. Careglio, "Energy efficiency of elastic frequency grids in multilayer IP/MPLS-overflexgrid networks", J. of Netw. and Comp. Appl., vol. 56, pp. 41-47, 2015 (doi: 10.1016/j.jnca.2015.06.014).

[22] M. Fathy, S. GholamalitabarFirouzjaee, and K. Raahemifar, "Improving QoS in VANET Using MPLS", Procedia Comp. Science, vol. 10, pp. 1018-1025, 2012 (doi: 10.1016/j.procs.2012.06.141).

[23] Z. Song, P. W. C. Prasad, A. Alsadoon, L. Pham, and A. Elchouemi, "Upgrading Internet service provider (ISP) network in multiprotocol label switching (MPLS) and border gateway protocol (BGP) environment", in Proc. Int. Conf. on Adv. in Elec., Electron. and Syst. Engin. ICAEES 2016, pp. 237-241, Putrajaya, Malaysia, 2016 (doi: 10.1109/ICAEES.2016.7888045).

[24] B. Genge and C. Siaterlis, "Analysis of the effects of distributed denial-of-service attacks on MPLS networks", Int. J. of Crit. Infrastruc. Protect., vol. 6, no. 2, pp. 87-95, 2013 (doi: 10.1016/j.ijcip.2013.04.001).

[25] M. N. Soorki and H. Rostami, "Label switched protocol routing with guaranteed bandwidth and end to end path delay in MPLS networks", J. of Netw. and Comp. Appl., vol. 42, pp. 21-38, 2014 (doi: 10.1016/j.jnca.2014.03.008).

[26] G. A. Mazhin, M. Bag-Mohammadi, M. Ghasemi, and S. Feizi, "Multi-layer architecture for realization of network virtualization using MPLS technology", ICT Express, vol. 3, no. 1, pp. 43-47, 2017 (doi: 10.1016/j.icte.2016.07.002).

[27] F. Francois, N. Wang, K. Moessner, S. Georgoulas, and R. de Oiveira Schmidt, "Leveraging MPLS backup paths for distributed energyaware traffic engineering", IEEE Trans. on Netw. and Serv. Manag. vol. 11, no. 2, pp. 235-249, 2014 (doi: 10.1109/TNSM.2014.2321839).

[28] L. Andersson, A. B. Acreo, and R. Asati, "Multiprotocol Label Switching (MPLS) Label Stack Entry: "EXP" Field Renamed to "Traffic Class" Field", RFC 5462, IETF, 2009 [Online]. Available: https://tools.ietf.org/html/rfc5462

[29] L. Wu et al., "Multi-Protocol Label Switching (MPLS) Support of Differentiated Services", RFC 3270, IETF, 2002 [Online]. Available: https://tools.ietf.org/html/rfc3270

[30] N. Rouhana and E. Horlait, "Differentiated services and integrated services use of MPLS", in Proc. 5th IEEE Symp. on Comp. and Commun. ISCC 2000., Antibes-Juan Les Pins, France, 2000, pp. 194-199 (doi: 10.1109/ISCC.2000.860638).

[31] J. Heinanen, F. Baker, W. Weiss, and J. Wroclawski, "Assured Forwarding PHB Group", RFC 2597, IETF, 1999 [Online]. Available: https://tools.ietf.org/html/rfc2597

[32] R. Pletka, P. Droz, and B. Stiller, "A Buffer-Management Scheme for Bandwidth and Delay Differentiation Using a Virtual Scheduler", in Networking - ICN 2001: First International Conference on Networking Colmar, France, July 9-13, 2001 Proceedings, Part I, P. Lorenz, Ed. LNCS, vol. 2093, pp. 218-234. Berlin, Heidelberg: Springer, 2001.

[33] R. Balakrishnan, Advanced QoS for Multi-Service IP/MPLS Networks. Wiley, 2012 (ISBN: 9781118621479).

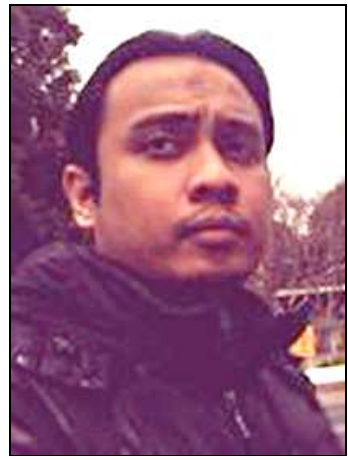

Addie Irawan has been a Senior Lecturer at the Universiti Malaysia Pahang (UMP), Pahang, Malaysia since 2005, working at the Faculty of Electrical and Electronic Engineering (FKEE). His areas of interest include robotics, dynamic and motion control, as well as computers and networks. He specializes in network protocols 
and big data distributions. He is a Professional Engineer of the Board of Engineers Malaysia (BEM), a Charted Engineer and a Charted Marine Engineer under the British Engineering Council via the Institute of Marine Engineering Science and Technology (IMarEST), as well as a Senior Member of the Institute of Electrical and Electronics Engineers (IEEE).

E-mail: addieirawan@ump.edu.my

Robotics and Unmanned Systems (RUS) research group Faculty of Electrical and Electronics Engineering

Universiti Malaysia Pahang

26600 Pekan, Pahang, Malaysia

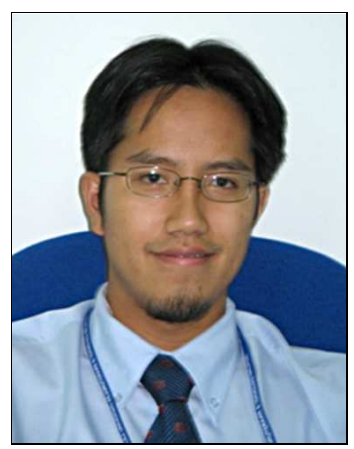

Mohammad Fadhil Abas received his B.Sc. in Electric, Electronic and Computer Systems form Universiti Kebangsaan Malaysia, Malaysia in 2002, his M.Sc. in Power Electronics from University Putra Malaysia, Malaysia in 2005 and his Ph.D. in Artificial Science from Chiba University, Japan in 2013. Since 2004 he has been working at Universiti Malaysia Pahang (UMP), as a Senior Lecturer at the Faculty of Electrical and Electronic Engineering. In his work, he focuses mainly on the following areas: instrumentation, humancomputer interface, human-robot interface, unmanned sys- tems, automation, vision systems for robotics and control systems.

E-mail: mfadhil@ump.edu.my

Robotics and Unmanned Systems (RUS) research group

Faculty of Electrical and Electronics Engineering

Universiti Malaysia Pahang

26600 Pekan, Pahang, Malaysia

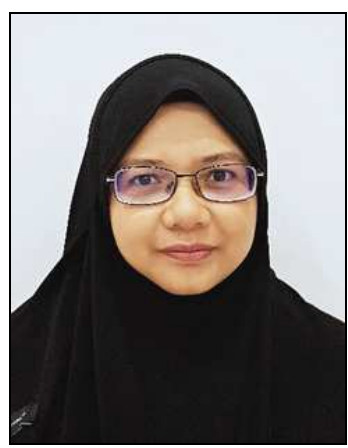

Nurulfadzilah Hasan received her B.Sc. degree in Computer Engineering from Universiti Teknologi Malaysia in 2003. She received her M.Sc. degree in Electrical Engineering from the same university in 2005. She has been working as a lecturer at Universiti Malaysia $\mathrm{Pa}$ hang since 2006, where she has been involved in several research projects and has published numerous research papers. Her research areas include antenna design, wireless communications and computer engineering. Presently, she is pursuing her Ph.D. in antenna design at Universiti Malaysia Pahang.

E-mail: nurulfadzilah@ump.edu.my

Applied Electronics (AE) research group

Faculty of Electrical and Electronics Engineering

Universiti Malaysia Pahang

26600 Pekan, Pahang, Malaysia 